\title{
A lack of association between vitamin D-binding protein and 25-hydroxyvitamin D concentrations in pediatric type 1 diabetes without microalbuminuria
}

\author{
Hwa Young Kim, MD', \\ Young Ah Lee, MD, PhD', \\ Hae Woon Jung, $\mathrm{MD}^{3}$, \\ Min Jeoung $\mathrm{Gu}^{2}$, \\ Ji Young Kim², \\ Gyung Min Lee, MD', \\ Jieun Lee, $M D^{5}$, \\ Ju Young Yoon, $\mathrm{MD}^{6}$, \\ Sei Won Yang, MD, PhD', \\ Choong Ho Shin, MD, PhD ${ }^{2}$
}

${ }^{1}$ Department of Pediatrics, Kangwon National University Hospital, Chuncheon, ${ }^{2}$ Department of Pediatrics, Seoul National University Children's Hospital, Seoul, ${ }^{3}$ Department of Pediatrics, Kyung Hee University Medical Center, Seoul, ${ }^{4}$ Department of Pediatrics, Konyang University Hospital, Daejeon, ${ }^{5}$ epartment of Pediatrics, Inje University Ilsan Paik Hospital, Inje University College of Medicine, Goyang, ${ }^{6}$ Gyeongsang National University Hospital, Changwon, Korea

Received: 28 March, 2017

Revised: 2 June, 2017

Accepted: 26 June, 2017

Address for correspondence:

Choong Ho Shin, MD, PhD

Department of Pediatrics, Seoul National University Children's Hospital, 101 Daehak-ro, Jongno-gu, Seoul 03080, Korea

Tel: +82-2-2072-3357

Fax: +82-2-743-3455

E-mail: chshinpd@snu.ac.kr

https://orcid.org/0000-0002-98131134
Purpose: Vitamin $D$ deficiency is reported to be more common in type 1 diabetes patients and might be associated with the increased urinary loss of vitamin $D$ binding protein (VDBP) consequent to impaired 25-hydroxyvitamin D (25(OH)D) circulation. We aimed to evaluate the possible increased urinary loss of VDBP, a correlation between VDBP and circulating 25(OH)D level, and risk factors influencing low vitamin $D$ level in pediatric type 1 diabetes patients without microalbuminuria. Methods: This is a cross-sectional study of subjects who visited Seoul National University Children's Hospital between January and March 2013. Forty-two type 1 diabetes patients and 29 healthy controls were included. Biochemical parameters including serum and urine VDBP concentrations were analyzed.

Results: There was no significant difference in the frequency of vitamin D deficiency or serum 25(OH)D level between the 2 groups. The serum and urine VDBP concentrations did not show any difference between the 2 groups. Serum $25(\mathrm{OH})$ $D$ level did not correlate with serum or urine VDBP. Multivariate regression analysis revealed that daylight outdoor hours $(\beta=2.948, P=0.003)$ and vitamin $D$ intake $(\beta=2.865, P=0.003)$ affected the $25(\mathrm{OH}) \mathrm{D}$ level; the presence of type 1 diabetes or urinary VDBP excretion was not significant.

Conclusion: In pediatric type 1 diabetes patients, urinary VDBP excretion did not contribute to low serum $25(\mathrm{OH}) \mathrm{D}$ level in the setting of normoalbuminuria. The factors associated with $25(\mathrm{OH}) \mathrm{D}$ level during winter periods were daylight outdoor hours and vitamin D intake. Further studies including both micro- and macroalbuminuria patients with type 1 diabetes are warranted.

Keywords: Vitamin D-binding protein, Ergocalciferols, Type 1 diabetes mellitus, Child, Albuminuria

\section{Introduction}

Several potential causative mechanisms of the reduced bone mineral density associated with type 1 diabetes in adults have been proposed, including bone changes due to advanced glycation, hypercalciuria associated with glycosuria, increased inflammatory cytokine levels, and a high prevalence of vitamin $\mathrm{D}$ deficiency ${ }^{1}$. Many studies have suggested that vitamin $\mathrm{D}$ deficiency is more common in individuals with type 1 diabetes, compared with controls ${ }^{1,22}$, but the mechanisms that contribute to vitamin $\mathrm{D}$ deficiency in type 1 diabetes patients remain unclear $^{3}$. Currently, the importance of a sufficient vitamin D status has been emphasized by the discovery of nonclassic actions of vitamin D on insulin resistance, cardiovascular diseases, cancer, and others ${ }^{4.5)} .25$-Hydroxyvitamin D (25(OH)D), the major circulating form of vitamin $\mathrm{D}$, is the best indicator with which to monitor vitamin D status. Approximately $88 \%$ of serum $25(\mathrm{OH}) \mathrm{D}$ is bound to the vitamin D-binding protein (VDBP), a major vitamin D metabolite 
transport protein in the plasma ${ }^{6}$. The VDBP $+25(\mathrm{OH}) \mathrm{D}$ complex is filtered across the glomerulus and transported to the proximal tubule. Next, reabsorption of the VDBP $+25(\mathrm{OH})$ D complex by the multiligand receptors, megalin and cubilin, promotes the conversion of $25(\mathrm{OH}) \mathrm{D}$ to 1,25 -dihydroxyvitamin $\mathrm{D}\left(1,25(\mathrm{OH})_{2} \mathrm{D}\right)$ through the activity of $1 a$-hydroxylase $\mathrm{e}^{3,7,8)}$. The risk of vitamin $\mathrm{D}$ deficiency might increase along with an increased loss of urine VDBP (uVDBP) as a result of impaired $25(\mathrm{OH}) \mathrm{D}$ recirculation. In a study of type 1 diabetes patients, aged 14-40 years, with a disease duration of approximately 10 years, the excretion of uVDBP was reported to be exaggerated relative to the degree of albuminuria, and the frequency of vitamin $\mathrm{D}$ deficiency was found to be higher in albuminuric patients ${ }^{3}$. However, there have been no studies among pediatric patients with short disease durations and no albuminuria. In this study, we examined whether there would be an increased loss of uVDBP in pediatric type 1 diabetes patients without microalbuminuria and whether a correlation would exist between VDBP levels and vitamin D deficiency or circulating $25(\mathrm{OH}) \mathrm{D}$ levels. We also aimed to identify the risk factors that influenced low vitamin D levels in pediatric type 1 diabetes patients.

\section{Materials and methods}

\section{Subjects}

Patients with type 1 diabetes without microalbuminuria $(n=42)$ and healthy control subjects $(n=29)$, aged 9-14 years, were included in this hospital-based cross-sectional study in the capital region of Seoul $\left(37^{\circ} \mathrm{N}\right)$, South Korea. The subjects were recruited from the pediatric endocrinology outpatient clinic at Seoul National University Children's Hospital. This study was conducted from January to March 2013 to exclude any seasonal variations. The exclusion criteria included a history of current or previous use of medications or medical conditions known to affect vitamin D metabolism or bone health, a history of malignancy or other autoimmune disease, restricted physical activity, short stature ( $<3$ rd percentile), obesity (body mass index $[\mathrm{BMI}]>95$ th percentile), or orthostatic proteinuria.

\section{Measurements}

The height, weight, and waist circumference were measured and pubertal status evaluated in all subjects. Height was measured with a Harpenden stadiometer (Holtain Ltd., Crymych, Wales, UK), and weight was measured with a digital scale. The BMI was calculated as the weight $(\mathrm{kg})$ divided by the height squared $\left(\mathrm{m}^{2}\right)$. The minimal and umbilical waist circumferences were measured. The $Z$-scores of height and BMI were assigned according to the 2007 Korean National Growth Charts" ${ }^{9}$. Pubertal onset was defined as breast budding in females and increased testicular volume $(\geq 4 \mathrm{~mL})$ in males. Bone age was assessed according to the Greulich and Pyle method.
The body fat percentage was measured using bioelectrical impedance analysis, Inbody 3.0 (Biospace Ltd., Seoul, Korea). Vitamin D deficiency was defined as a $25(\mathrm{OH}) \mathrm{D}$ level of $<20$ $\mathrm{ng} / \mathrm{mL}^{6}$. The family history as well as previous medical and medication histories were evaluated using a questionnaire. A trained dietician collected information regarding vitamin $\mathrm{D}$ intake using a food-frequency questionnaire. The vitamin D content of foods were determined from the Rural Development Administration food composition tables ${ }^{10)}$, and the vitamin D contents of supplements were also identified. A questionnaire was used to assess the amount and intensity of physical activity including daylight outdoor hours and sedentary hours. The subjects' levels of physical activity were investigated during a normal week. The definition of regular physical activity was based on the physical activity guidelines of the United States Department of Health and Human Services ${ }^{11}$. Subjects with physical activity (moderate or vigorous-intensity physical activity) of at least $60 \mathrm{~min} /$ day for 7 days per week were considered to have participated in regular physical activity.

Serum levels of calcium, phosphorus, alkaline phosphatase, $25(\mathrm{OH}) \mathrm{D}, 1,25(\mathrm{OH})_{2} \mathrm{D}$ were measured. Whole blood was obtained in ethylenediaminetetraacetic acid (EDTA) and sodium hepatin cell preparation tubes for quantification of intact parathyroid hormone (PTH) and serum VDBP (sVDBP). EDTA tubes were centrifuged at $1,000 \times \mathrm{g}$ for 15 minutes within 30 minutes of collection and stored at $-70^{\circ} \mathrm{C}$ for analysis. A random urine sample was used to measure the microalbumin and creatinine $(\mathrm{Cr})$. Random urine samples for uVDBP were stored at $-70^{\circ} \mathrm{C}$. The serum intact PTH level was measured using the standard ELSA-PTH immunoradiometric assay (CIS Bio International, Sorgues, France) with an interassay coefficient of variation (CV) of $4.6 \%$ and an intra-assay $\mathrm{CV}$ of $4.3 \%$. The serum $25(\mathrm{OH}) \mathrm{D}$ level was measured using an ${ }^{125}$ I-labeled radioimmunoassay (DiaSorin, Stillwater, MN, USA) with an interassay CV of $10.8 \%$ and an intra-assay CV of $9.4 \%$. The serum $1,25(\mathrm{OH}) 2 \mathrm{D}$ level was measured using an ${ }^{125}$ I-labeled radioimmunoassay (DiaSorin) with an interassay $\mathrm{CV}$ of $8.6 \%$ and an intra-assay CV of $13.8 \%$. The VDBP levels were measured in duplicate by commercial enzyme-linked immunosorbent assay (Quantikine ELISA no. DVDBP0; R\&D Systems, Minneapolis, MN, USA) according to the manufacturer's instructions with a normal range of serum 56 to $473 \mu \mathrm{g} / \mathrm{mL}$ and urine $0-250 \mathrm{ng} / \mathrm{mL}$. The intra- and interassay coefficients of variation for this assay were $5.9 \%$ and $6.2 \%$, respectively. The urine microalbumin and $\mathrm{UVDBP}$ values were normalized to the urine $\mathrm{Cr}$ concentrations and expressed as a microalbumin to Cr ratio ( $\mathrm{mACR}, \mathrm{mg} / \mathrm{g}$ ) and as a uVDBP to Cr ratio (uVDBPCR, ng/g), respectively, to control for variable urine concentrations. Normoalbuminuria was defined as $\mathrm{mACR} \leq 30 \mathrm{mg} / \mathrm{g}$.

\section{Statistical analysis}

Statistical analyses were performed with IBM SPSS ver. 18.0 
(IBM Co., Armonk, NY, USA). All continuous variables were described as mean \pm standard deviation. The variables were tested for normal distributions. Vitamin D intake, daylight outdoor hours (hr/wk), sVDBP, uVDBPCR, and mACR had skewed distributions and were therefore log-transformed for the analysis. Student t-test was used to compare the mean values, and the chi-square test was used to compare categorical variables between 2 groups with normally distributed data. Pearson correlation coefficients were assessed as the measure of correlation between variables of interest. A univariate linear regression analysis was performed to identify the risk factors of a low 25(OH)D level. A multivariate regression analysis was subsequently performed and included all significant independent variables from the univariate analysis and the previously known covariates (sex, body fat percentage, presence of type 1 diabetes, and the uVDBPCR). For all analyses, a $P$-value $<0.05$ was considered significant.

\section{Ethics Statement}

The study protocol was approved by the Institutional Review Board of Seoul National University Hospital (approval number:
H-1211-083-442). Written informed consent was obtained from a parent or guardian as well as the subjects.

\section{Results}

\section{Baseline participant characteristics}

The patients with type 1 diabetes were older than those in the control group $(12.2 \pm 1.8$ years for patients with type 1 diabetes vs. $11.0 \pm 1.3$ years for controls, $P=0.002$ ). There were no differences with regard to sex, pubertal status, height $Z$-score, BMI $Z$-score, and body fat percentage between the 2 groups. In the patients with type 1 diabetes, the disease duration was $6.4 \pm 3.0$ years (range, $1.1-12.0$ years), and the hemoglobin Alc level was $8.6 \% \pm 1.2 \%$. There were no differences with regard to vitamin $\mathrm{D}$ intake, daylight outdoor hours, regular physical activity and frequency of sunscreen use between the groups. Patients with type 1 diabetes had more sedentary hours $(P=0.004)$ than the controls (Table 1).

Table 1. Demographic characteristics of the study subjects

\begin{tabular}{|c|c|c|c|}
\hline Characteristic & Control $(n=29)$ & Type 1 diabetes $(n=42)$ & $P$-value \\
\hline Male sex & $13(44.8)$ & $12(28.6)$ & 0.159 \\
\hline Chronological age (yr) & $11.0 \pm 1.3$ & $12.2 \pm 1.8$ & 0.002 \\
\hline Bone age (yr) & $11.7 \pm 1.7$ & $12.8 \pm 2.1$ & 0.010 \\
\hline Puberty & $20(69.0)$ & $35(83.3)$ & 0.154 \\
\hline Height Z-score & $0.03 \pm 0.9$ & $0.23 \pm 1.1$ & 0.435 \\
\hline BMI Z-score & $0.06 \pm 0.8$ & $-0.12 \pm 0.8$ & 0.377 \\
\hline Body fat (\%) & $23.6 \pm 6.4$ & $23.1 \pm 7.0$ & 0.815 \\
\hline Total vitamin D intake ( $\mu \mathrm{g} /$ day) $)^{*}$ & $8.1 \pm 6.9$ & $9.9 \pm 8.4$ & 0.429 \\
\hline Sunscreen use & $3(10.3)$ & $9(21.4)$ & 0.221 \\
\hline Daylight outdoor hours (hr/wk) & $11.2 \pm 8.1$ & $16.3 \pm 14.3$ & 0.232 \\
\hline Regular physical activity & $3(10.3)$ & $6(14.3)$ & 0.624 \\
\hline Sedentary hours $(\mathrm{hr} / \mathrm{wk})^{\dagger}$ & $15.0 \pm 10.4$ & $22.7 \pm 10.8$ & 0.004 \\
\hline
\end{tabular}

Values are presented as number (\%) or mean \pm standard deviation.

*Natural log transformed values were used for the analysis. ${ }^{\dagger}$ Hours of watching television or a computer.

Table 2. Comparison of metabolic characteristics between the control and type 1 diabetes groups

\begin{tabular}{lccc}
\hline Variable & Control $(\mathrm{n}=29)$ & Type 1 diabetes $(\mathrm{n}=42)$ & $P$-value \\
\hline 25(OH)D deficiency & $13(44.8)$ & $24(57.1)$ & 0.307 \\
25(OH)D $(\mathrm{ng} / \mathrm{mL})$ & $21.3 \pm 6.0$ & $20.0 \pm 6.4$ & 0.426 \\
Serum VDBP $(\mu \mathrm{g} / \mathrm{mL})^{*}$ & $175.0 \pm 84.6$ & $200.8 \pm 75.2$ & 0.108 \\
Urine VDBP $(\mathrm{ng} / \mathrm{mL})^{*}$ & $34.0 \pm 31.0$ & $48.2 \pm 35.9$ & 0.127 \\
Urine VDBP/Cr $(\mathrm{ng} / \mathrm{mg})^{*}$ & $55.4 \pm 49.3$ & $72.6 \pm 53.3$ & 0.305 \\
Urine microalbumin/Cr $(\mathrm{mg} / \mathrm{g})^{*}$ & $11.1 \pm 7.5$ & $14.0 \pm 6.2$ & 0.012 \\
Total calcium $(\mathrm{mg} / \mathrm{dL})$ & $9.6 \pm 0.4$ & $9.6 \pm 0.3$ & 0.920 \\
Phosphorus $(\mathrm{mg} / \mathrm{dL})$ & $4.9 \pm 0.5$ & $5.0 \pm 0.8$ & 0.545 \\
Alkaline phosphatase $(\mathrm{IU} / \mathrm{L})$ & $224.7 \pm 73.4$ & $249.2 \pm 107.5$ & 0.257 \\
Parathyroid hormone $(\mathrm{pg} / \mathrm{mL})$ & $22.7 \pm 12.7$ & $22.3 \pm 11.4$ & 0.902 \\
\hline Values are & &
\end{tabular}

Values are presented as number (\%) or mean \pm standard deviation.

25(OH)D, 25-hydroxyvitamin D; VDBP, vitamin D-binding protein; $\mathrm{Cr}$, creatinine.

*Natural log transformed values were used for the analysis. 


\section{Biochemical parameters of the participants}

There was no significant difference in the frequency of vitamin D deficiency during the winter season (44.8\% for controls vs. $57.1 \%$ for patients with type 1 diabetes). The serum $25(\mathrm{OH}) \mathrm{D}$ levels did not show any differences between the 2 groups. The sVDBP and uVDBPCR did not show any difference between the 2 groups. The urine mACR was significantly higher in the patients with type 1 diabetes than in the control group $(P=0.012)$. There were no differences in serum levels of calcium, phosphorus, alkaline phosphatase and PTH between the groups (Table 2).

\section{Association between VDBP and serum 25(OH)D levels or vitamin $\mathrm{D}$ deficiency}

The sVDBP and uVDBPCR levels did not differ between the vitamin $\mathrm{D}$ deficiency and nondeficiency groups (Table 3). The uVDBPCR positively correlated with $\mathrm{mACR}$ in both groups (control: $r=0.400, P=0.031$; type 1 diabetes: $r=0.614, P<0.001$ ). The $25(\mathrm{OH}) \mathrm{D}$ level did not correlate with the sVDBP or uVDBPCR in both groups.

\section{Factors associated with low serum $25(\mathrm{OH}) \mathrm{D}$ level}

We performed univariate analyses to determine the risk factors of a low serum 25(OH)D level, using the serum $25(\mathrm{OH})$ $D$ levels as the outcome variable. Low total vitamin $D$ intake $(P=0.005)$, older age $(P=0.039)$ and reduced daylight outdoor hours $(P=0.015)$ were significantly associated with a low vitamin
D status (Table 4). A multivariate regression analysis including the known risk factors for vitamin D deficiency such as age, sex, body fat percentage, vitamin $\mathrm{D}$ intake, daylight outdoor hours, presence of type 1 diabetes, and the $\mathrm{UVDBPCR}{ }^{3,12,13)}$ revealed that both the presence of type 1 diabetes and $\mathrm{UVDBPCR}$ were not risk factors for vitamin $\mathrm{D}$ deficiency. The factors that affected 25(OH)D levels were daylight outdoor hours $(\beta=2.948$, $P=0.003)$ and total vitamin $\mathrm{D}$ intake $(\beta=2.865, P=0.003)$ (Table $4)$.

\section{Discussion}

The prevalence of vitamin D deficiency in pediatric patients with type 1 diabetes without microalbuminuria during the winter season (57.1\%) was not different from a normal control group. The sVDBP and uVDBPCR did not show any difference between the 2 groups. The serum $25(\mathrm{OH}) \mathrm{D}$ level did not correlate with $\mathrm{uVDBPCR}$. In pediatric type 1 diabetes patients with normoalbuminuria, the factors that affected serum $25(\mathrm{OH})$ $D$ levels during the winter period were daylight outdoor hours and the vitamin D intake. The uVDBPCR did not correlate with the serum 25(OH)D levels.

In our study, uVDBPCR showed positive correlation with $\mathrm{mACR}$ as in the previous studies ${ }^{3,14}$. However, the uVDBPCR did not show any difference between the 2 groups in the setting of normoalbuminuria. VDBP is synthesized in hepatocytes and has a strong structural homology with albumin and a similar molecular weight ( $69 \mathrm{kDa}$ for albumin vs. $58 \mathrm{kDa}$ for VDBP ${ }^{15,16)}$. Furthermore, both albumin and VDBP uptake in the proximal tubule are principally controlled by megalin and

Table 3. Comparison of vitamin D and related analytes according to vitamin D status

\begin{tabular}{|c|c|c|c|c|c|c|}
\hline \multirow{2}{*}{ Variable } & \multicolumn{3}{|c|}{ Control } & \multicolumn{3}{|c|}{ Type 1 diabetes } \\
\hline & $\operatorname{VDD}(n=13)$ & $N D(n=16)$ & $P$-value & $\operatorname{VDD}(n=24)$ & $N D(n=18)$ & $P$-value \\
\hline $25(\mathrm{OH}) \mathrm{D}(\mathrm{ng} / \mathrm{mL})$ & $15.8 \pm 2.6$ & $25.7 \pm 3.9$ & $<0.001$ & $15.4 \pm 3.4$ & $26.2 \pm 3.5$ & $<0.001$ \\
\hline Serum VDBP $(\mu \mathrm{g} / \mathrm{mL})^{*}$ & $172.3 \pm 78.1$ & $177.2 \pm 92.0$ & 0.998 & $205.6 \pm 71.3$ & $194.2 \pm 81.7$ & 0.478 \\
\hline Urine VDBP $(\mu \mathrm{g} / \mathrm{mL})^{*}$ & $32.1 \pm 20.4$ & $35.5 \pm 38.2$ & 0.997 & $54.7 \pm 40.8$ & $39.7 \pm 26.7$ & 0.251 \\
\hline Urine VDBP/Cr (ng/mg) & $53.3 \pm 34.0$ & $57.1 \pm 60.1$ & 0.947 & $80.6 \pm 58.8$ & $62.0 \pm 44.3$ & 0.333 \\
\hline
\end{tabular}

Values are presented as mean \pm standard deviation.

VDD, vitamin D deficiency; ND, nondeficiency; 25(OH)D, 25-hydroxyvitamin D; VDBP, vitamin D-binding protein; $\mathrm{Cr}$, creatinine.

*Natural log transformed values were used for the analysis.

Table 4. Results of unadjusted and multivariate-adjusted linear regression analyses with 25-hydroxyvitamin D concentrations as the dependent variable in study subjects $(n=71)$

\begin{tabular}{|c|c|c|c|c|c|c|}
\hline \multirow{2}{*}{ Variable } & \multicolumn{3}{|c|}{ Unadjusted } & \multicolumn{3}{|c|}{ Multivariate-adjusted } \\
\hline & $\beta$ & SE & $P$-value & $\beta$ & SE & $P$-value \\
\hline Age & -0.890 & 0.423 & 0.039 & -0.655 & 0.460 & 0.160 \\
\hline Female sex & -1.894 & 1.543 & 0.224 & -0.970 & 1.593 & 0.545 \\
\hline Body fat & -0.146 & 0.110 & 0.191 & -0.093 & 0.117 & 0.430 \\
\hline Total vitamin D intake* & 2.721 & 0.940 & 0.005 & 2.865 & 0.923 & 0.003 \\
\hline Daylight outdoor hours ${ }^{*}$ & 2.435 & 0.974 & 0.015 & 2.948 & 0.953 & 0.003 \\
\hline Type 1 diabetes & -1.209 & 1.508 & 0.426 & -1.267 & 1.586 & 0.428 \\
\hline Urine $\mathrm{VDBP} / \mathrm{Cr}^{*}$ & -0.207 & 0.974 & 0.832 & -0.427 & 0.909 & 0.641 \\
\hline
\end{tabular}

SE, standard error; VDBP, vitamin D-binding protein; $\mathrm{Cr}$, creatinine.

"Natural log transformed values were used for the analysis. 
its coreceptor cubilin ${ }^{17)}$. Therefore, it seems that the VDBP and albumin go through similar process in the kidney. Regarding sVDBP, there was a report of decreased sVDBP levels in diabetic rats ${ }^{18)}$, and patients with type 1 diabetes have been found to have lower sVDBP levels when compared to controls ${ }^{19}$. However, according to a recent study, the sVDBP levels did not differ between patients with type 1 diabetes and the control group ${ }^{3)}$ as in our study.

There are not many studies which investigated the relationship between $\mathrm{uVDBP}$ and 25(OH)D levels ${ }^{3,20)}$. In a study conducted in diabetic DBA/2 J nephropathy-prone mice, urinary $25(\mathrm{OH}) \mathrm{D}$ excretion increased along with increasing uVDBP excretion ${ }^{21)}$. In albuminuric patients with type 1 diabetes, urinary megalin and cubilin excretion was increased, along with uVDBP ${ }^{22}$. Thrailkill et al. ${ }^{3)}$ reported that uVDBP excretion in type 1 diabetes patients aged 14-40 years was higher than that in the control group, and was further increased in patients with albuminuria. Furthermore, vitamin $\mathrm{D}$ deficiency and insufficiency were more common in patients with type 1 diabetes with albuminuria, coincident with the increase in $\mathrm{UVDBP}$ excretion. However, uVDBPCR did not associate with the serum $25(\mathrm{OH}) \mathrm{D}$ levels as in our study. This may be attributed to the small number of patients in the albuminuria group (9.4\%) and the even smaller number of macroalbuminuria patients $(1.18 \%)$ analyzed. Regarding the association between sVDBP and 25(OH)D level, most studies reported of no association ${ }^{14,19,23,24)}$ except one study reporting a positive correlation ${ }^{25}$. The VDBP and $25(\mathrm{OH}) \mathrm{D}$ levels may vary independently because VDBP $\left(5 \times 10^{-6} \mathrm{~mol} / \mathrm{L}\right)$ circulates at a concentration approximately 100 fold higher than that of its ligand $25(\mathrm{OH}) \mathrm{D}\left(5 \times 10^{-8} \mathrm{~mol} / \mathrm{L}\right)^{26}$. Only a severe and longterm loss of VDBP, as observed in nephrotic syndrome with continuous high-grade proteinuria, could lead to decreases in the 25(OH)D level ${ }^{20)}$.

Many studies found a higher prevalence of vitamin D deficiency in patients with type 1 diabetes when compared with the control group ${ }^{27-29)}$. In this study, we did not find a significant difference in the prevalence of vitamin D deficiency between the 2 groups. Because the frequency of vitamin D deficiency is high in healthy Korean children during the winter period ${ }^{30)}$, the prevalence of vitamin D deficiency might not differ between the type 1 diabetes and control groups during this season.

The factors known to contribute to low vitamin D levels are high latitude, winter season, black race, old age, female sex, high BMI, low physical activity, and low vitamin D intake ${ }^{12,13)}$. A study in type 1 diabetes reported season and the presence of type 1 diabetes as significant factors affecting vitamin D status $^{3}$. In this study, we excluded obese subjects and performed an analysis during the winter period at the same latitude to minimize the influence of ultraviolet rays, which comprise a significant factor with respect to the vitamin D status. In our study, although the patients with type 1 diabetes were older than those in the control group, there was no significant difference in the prevalence of vitamin D deficiency between the groups during the winter period. The daylight outdoor hours as well as vitamin D intake were the factor associated with the $25(\mathrm{OH})$ $\mathrm{D}$ level during the winter season. Stored vitamin $\mathrm{D}$, generated via outdoor activities during summer and autumn period, may affect vitamin $\mathrm{D}$ status in the winter. The presence of type 1 diabetes per se did not impair 25(OH)D circulation.

The limitations of our study include the cross-sectional design and relatively small number of patients. However, this is the first study to analyze the association between the VDBP level (serum and urine) and the $25(\mathrm{OH}) \mathrm{D}$ level or vitamin D deficiency in pediatric patients with type 1 diabetes without microalbuminuria. We also included all of the known risk factors such as the body fat percentage, sex, vitamin D intake, and daylight outdoor hours in the analysis while controlling for the effects of the season, latitude, and obesity.

In conclusion, there was no correlation between serum $25(\mathrm{OH}) \mathrm{D}$ and $\mathrm{uVDBP}$ level in the setting of normoalbuminuria without considerable uVDBP excretion. The factors associated with the $25(\mathrm{OH}) \mathrm{D}$ levels during the winter period were the daylight outdoor hours and vitamin D intake, not the presence of type 1 diabetes or uVDBPCR. Therefore, sufficient vitamin $D$ intake and daylight outdoor hours need to be emphasized in order to achieve improvements in vitamin D status.

\section{Conflict of interest}

No potential conflict of interest relevant to this article was reported.

\section{Acknowledgments}

This study was supported by grant from the Seoul National University College of Medicine Research Fund 2013.

\section{References}

1. Svoren BM, Volkening LK, Wood JR, Laffel LM. Significant vitamin $\mathrm{D}$ deficiency in youth with type 1 diabetes mellitus. J Pediatr 2009;154:132-4.

2. Littorin B, Blom P, Schölin A, Arnqvist HJ, Blohmé G, Bolinder J, et al. Lower levels of plasma 25-hydroxyvitamin $\mathrm{D}$ among young adults at diagnosis of autoimmune type 1 diabetes compared with control subjects: results from the nationwide Diabetes Incidence Study in Sweden (DISS). Diabetologia 2006;49:2847-52.

3. Thrailkill KM, Jo CH, Cockrell GE, Moreau CS, Fowlkes JL. Enhanced excretion of vitamin $\mathrm{D}$ binding protein in type 1 diabetes: a role in vitamin D deficiency? J Clin Endocrinol Metab 2011;96:142-9.

4. Bouillon R, Carmeliet G, Verlinden L, van Etten E, Verstuyf A, Luderer HF, et al. Vitamin D and human health: lessons from vitamin D receptor null mice. Endocr Rev 2008;29:726-76.

5. Bikle D. Nonclassic actions of vitamin D. J Clin Endocrinol Metab 2009;94:26-34. 
6. Holick MF, Binkley NC, Bischoff-Ferrari HA, Gordon CM, Hanley DA, Heaney RP, et al. Evaluation, treatment, and prevention of vitamin D deficiency: an Endocrine Society clinical practice guideline. J Clin Endocrinol Metab 2011;96:1911-30.

7. Nykjaer A, Dragun D, Walther D, Vorum H, Jacobsen C, Herz J, et al. An endocytic pathway essential for renal uptake and activation of the steroid 25- $(\mathrm{OH})$ vitamin D3. Cell 1999;96:507-15.

8. Dusso AS, Tokumoto M. Defective renal maintenance of the vitamin $\mathrm{D}$ endocrine system impairs vitamin $\mathrm{D}$ renoprotection: a downward spiral in kidney disease. Kidney Int 2011;79:715-29.

9. Korea Centers for Disease Control and Prevention, Division of Chronic Disease Surveillance, Committee for the Development of Growth Standard for Korean Children and Adolescents; Korean Pediatric Society, Committee for School Health and Public Health Statistics. 2007 Korean children and adolescents growth standard (commentary for the development of 2007 growth chart). Cheongju: Korea Centers for Disease Control and Prevention, Division of Chronic Disease Surveillance, 2007.

10. National Rural Resources Development Institute. Korean standard food composition table. 8th revision [Internet]. Wanju: National Institute of Agricultural Sciences; 2011 [cited 2014 Dec 10]. Available from: http://koreanfood.rda. go.kr/eng/fctFoodSrchEng/engMain.

11. U.S. Department of Health and Human Services. 2008 Physical activity guidelines for Americans [Internet]. Washington, DC: U.S. Department of Health and Human Services, c2008. [cited 2016 Jun 1]. Available from: http:// www.health.gov/paguidelines/guidelines.

12. Weaver CM. Vitamin D, calcium homeostasis, and skeleton accretion in children. J Bone Miner Res 2007;22 Suppl 2:V45-9.

13. Kumar J, Muntner P, Kaskel FJ, Hailpern SM, Melamed ML. Prevalence and associations of 25-hydroxyvitamin D deficiency in US children: NHANES 2001-2004. Pediatrics 2009; 124:e362-70.

14. Prytuła A, Wells D, McLean T, Balona F, Gullett A, Knott $\mathrm{C}$, et al. Urinary and dialysate losses of vitamin D-binding protein in children on chronic peritoneal dialysis. Pediatr Nephrol 2012;27:643-9.

15. Tojo A, Kinugasa S. Mechanisms of glomerular albumin filtration and tubular reabsorption. Int J Nephrol 2012;2012:481520.

16. Cooke NE, David EV. Serum vitamin D-binding protein is a third member of the albumin and alpha fetoprotein gene family. J Clin Invest 1985;76:2420-4.

17. Christensen EI, Birn H. Megalin and cubilin: multifunctional endocytic receptors. Nat Rev Mol Cell Biol 2002;3: 256-66.
18. Nyomba BL, Verhaeghe J, Thomasset M, Lissens W, Bouillon R. Bone mineral homeostasis in spontaneously diabetic BB rats. I. Abnormal vitamin D metabolism and impaired active intestinal calcium absorption. Endocrinology 1989; 124:565-72.

19. Blanton D, Han Z, Bierschenk L, Linga-Reddy MV, Wang H, Clare-Salzler M, et al. Reduced serum vitamin D-binding protein levels are associated with type 1 diabetes. Diabetes 2011;60:2566-70.

20. Alon US. Vitamin D metabolites in childhood nephrotic syndrome. Pediatr Nephrol 1995;9:791-2.

21. Fowlkes JL, Bunn RC, Cockrell GE, Clark LM, Wahl EC, Lumpkin CK, et al. Dysregulation of the intrarenal vitamin D endocytic pathway in a nephropathy-prone mouse model of type 1 diabetes. Exp Diabetes Res 2011;2011:269378.

22. Thrailkill KM, Nimmo T, Bunn RC, Cockrell GE, Moreau CS, Mackintosh S, et al. Microalbuminuria in type 1 diabetes is associated with enhanced excretion of the endocytic multiligand receptors megalin and cubilin. Diabetes Care 2009;32:1266-8.

23. Bolland MJ, Grey AB, Ames RW, Horne AM, Mason BH, Wattie DJ, et al. Age-, gender-, and weight-related effects on levels of 25-hydroxyvitamin $\mathrm{D}$ are not mediated by vitamin D binding protein. Clin Endocrinol (Oxf) 2007;67:259-64.

24. Winters SJ, Chennubhatla R, Wang C, Miller JJ. Influence of obesity on vitamin D-binding protein and 25-hydroxy vitamin D levels in African American and white women. Metabolism 2009;58:438-42.

25. Carpenter TO, Zhang JH, Parra E, Ellis BK, Simpson C, Lee WM, et al. Vitamin D binding protein is a key determinant of 25-hydroxyvitamin D levels in infants and toddlers. J Bone Miner Res 2013;28:213-21.

26. Speeckaert M, Huang G, Delanghe JR, Taes YE. Biological and clinical aspects of the vitamin $\mathrm{D}$ binding protein (Gc-globulin) and its polymorphism. Clin Chim Acta 2006;372:33-42

27. Hamed EA, Faddan NH, Elhafeez HA, Sayed D. Parathormone--25(OH)-vitamin D axis and bone status in children and adolescents with type 1 diabetes mellitus. Pediatr Diabetes 2011;12:536-46.

28. Chakhtoura M, Azar ST. The role of vitamin d deficiency in the incidence, progression, and complications of type 1 diabetes mellitus. Int J Endocrinol 2013;2013:148673.

29. Greer RM, Portelli SL, Hung BS, Cleghorn GJ, McMahon SK, Batch JA, et al. Serum vitamin D levels are lower in Australian children and adolescents with type 1 diabetes than in children without diabetes. Pediatr Diabetes 2013;14:31-41.

30. Lee YA, Kim HY, Hong H, Kim JY, Kwon HJ, Shin CH, et al. Risk factors for low vitamin D status in Korean adolescents: the Korea National Health and Nutrition Examination Survey (KNHANES) 2008-2009. Public Health Nutr 2014;17:764-71. 